\title{
Opinion Prediction with User Fingerprinting
}

\author{
Kishore Tumarada $^{1}$, Dr. Yifan Zhang1, Dr. Fan Yang ${ }^{2}$, \\ Dr. Eduard Dragut ${ }^{3}$, Dr. Omprakash Gnawali ${ }^{1}$, and Dr. Arjun Mukherjee ${ }^{1}$ \\ ${ }^{1}$ Department of Computer science, University of Houston, USA \\ ${ }^{2}$ Amazon, USA \{The paper was done before the author joined Amazon.\} \\ ${ }^{3}$ Department of Computer and information systems, Temple University, USA \\ $\{k i s h o r e . t 04$, aeryen, fyangskip\}@gmail.com, \\ edragutetemple.edu, \{odgnawal, amukher6\}@central.uh.edu
}

\begin{abstract}
Opinion prediction is an emerging research area with diverse real-world applications, such as market research and situational awareness. We identify two lines of approaches to the problem of opinion prediction. One uses topicbased sentiment analysis with time-series modeling, while the other uses static embedding of text. The latter approaches seek user-specific solutions by generating user fingerprints. Such approaches are useful in predicting user's reactions to unseen content. In this work, we propose a novel dynamic fingerprinting method that leverages contextual embedding of user's comments conditioned on relevant user's reading history. We integrate BERT variants with a recurrent neural network to generate predictions. The results show up to $13 \%$ improvement in micro F1-score compared to previous approaches. Experimental results show novel insights that were previously unknown such as better predictions for an increase in dynamic history length, the impact of the nature of the article on performance, thereby laying the foundation for further research.
\end{abstract}

\section{Introduction}

Sentiment analysis plays a key role in economic, social, and political contexts. Companies can understand customer's opinions based on reviews and/or social media conversations to make fast and accurate product decisions. They can index unstructured customer data at scale based on broad sentiments such as positive, negative, or neutral. It also enables market research - explore new markets, anticipate future trends, and seek an edge on the competition. In a political context, sentiment analysis can be useful in understanding political homophily using tweets analysis (Caetano et al., 2018).

Moreover, as social media has emerged as a new source of communication, governments can analyze people's reactions to issues such as police- related encounters, mob protests and anticipate responses before they turn violent. In this context, predicting people's opinion before expressing is an important next step in applying sentiment analysis to real-world applications. We are approaching this problem through sentiment prediction, especially on current news events, that can raise situational awareness, understand the future viewpoints of the citizenry on pressing social and political issues.

Recently fine-grained models generated a person's fingerprint, based on one's recent reading and response history, to predict response on an unknown event (Yang et al., 2020). In this work, we propose a novel architecture to create a dynamic fingerprint of a user that is contingent upon the target event. We choose to evaluate our models on the dataset used by Yang et al., 2020, which contains newspaper articles as events and users' comments on them as opinions. Those ground truth opinions are used as a basis for sentiment prediction in this work.

Our model consists of three main steps. In the first step, relevant articles are extracted from a user's reading history based on their similarity to target article. In the second step, the contextual embedding of these relevant articles, conditioned on the target article, is used to create a reading track. Similarly, we create a writing (response) track with the contextual embedding of extracted articles with corresponding comments by the user. Lastly, dynamic fingerprints are generated based on the temporal pattern of the reading and writing tracks. These dynamic fingerprint vectors for a particular user are then used to predict the user's sentiment on the target event.

Our main contributions in this paper are:

1. A novel architecture of dynamic fingerprint generation based on the contextual embedding of the user's reading history.

2. Experimental results show that our method 
outperformed the previous approach over various news outlets datasets.

\section{Related work}

Opinion prediction based on the temporal pattern of sentiments is a relatively new research topic, but basic concepts such as sentiment analysis, question answering based on dialogue context have been explored in different communities and settings.

Si et al., 2013 proposed a technique to leverage topic-based Twitter sentiments to predict the stock market using vector autoregression and Dirichlet process mixture models. Li et al., 2019 proposed a time+user dual attention-based LSTM network to perform emotional analysis on Chinese public opinion texts on social networking platform. But they did not use contextual embedding and explore the prospect of generating a unique user fingerprint before predicting sentiment.

Conversational question answering (CQA) is an emerging research area in the machine reading comprehension task (MRC). For single-turn MRC tasks, contextualized language representation using BERT has obtained state-of-the-art scores on SQuAD datasets (Devlin et al., 2019). CQA is a multi-turn question answering task that includes passage comprehension, contextual understanding, and coreference resolution. Zhu et al. have proposed SDNet (Zhu et al., 2018) to solve this problem by concatenating previous questions and answers as one query to fuse context into traditional MRC models by leveraging BERT, attention, and RNN techniques. Similarly, Ohsugi et al., 2019 have proposed fine-tuning approach with BERT in a multi-turn context by modeling the interaction between paragraph and dialogue history independently.

However, these models cannot be applied to the present problem since they did not integrate the concepts of the sequential pattern of sentiments along with the unique fingerprint of each user, which can play a key role in predicting the future opinion of a user on different topics.

\section{Proposed model and methodology}

In this section, we propose two classes of FingerPrint Embedding models (FPE) - Static and Dynamic - for the task of predicting the sentiment of a user $u$ to a new article. In a narrow sense, we used the term static to refer to the approach of using recent comment history, which is independent of the nature of target article $\mathrm{A}(\mathrm{t})$, and dynamic to refer to the approach of using articles relevant to target article A(t) in the overall history of user's comments, which are dependent on the nature of target article. In a broad sense, static and dynamic terms distinguish the way target article $\mathrm{A}(\mathrm{t})$ is integrated with user's reading history to generate the fingerprint.

We used the user's commenting history on articles that they read. We assume that we know the sentiment of each comment. (This can be obtained with one of the many sentiment analysis tools.) Formally, we are given the articles, comments along with the sentiments of a user $u$, i.e., $\left(A_{1}, C_{1}, S_{1}\right),\left(A_{1}, C_{2}, S_{2}\right), \ldots,\left(A_{2}, C_{j}, S_{j}\right), \ldots$, $\left(A_{t-1}, C_{n}, S_{n}\right)$, and the goal is to predict the sentiment $S_{t}$ of $u$ 's response to unseen article $A_{t}$. In general, $n>t$ because a user may post multiple comments to an article.

The overall architecture includes history selection, text embedding, fingerprint creation, and lastly sentiment prediction. We describe them below.

\subsection{History selection}

We have explored two methods of history selection - static history and dynamic history.

Given an article $A_{t}$, its static history, according to the user $u$, is the list of the most recent articlecomment pairs posted by $u$. Depending on the magnitude of $s$ and number of comments made by the user, the list may include comments from one article or multiple articles. We use this method in the Static FPE model.

For the dynamic history of article $A_{t}$, we have ranked all articles of author's reading history based on similarity and picked top $r$ articles, along with their comments, as shown in Figure 1. Here similarity between articles is calculated using DistilRoBERTa Semantic textual similarity model (Reimers and Gurevych, 2019).

It is a variant of sentence-BERT, a modified pretrained BERT network that uses siamese and triplet network structures to derive semantically meaningful sentence embeddings that are then compared using the cosine-similarity metric.

Table 1 shows the article samples extracted by both methods. For the target article "Pelosi says house will condemn all hate as anti-semitism debate overshadows congress", we can see that articles in the dynamic history, especially articles 1,3,4 


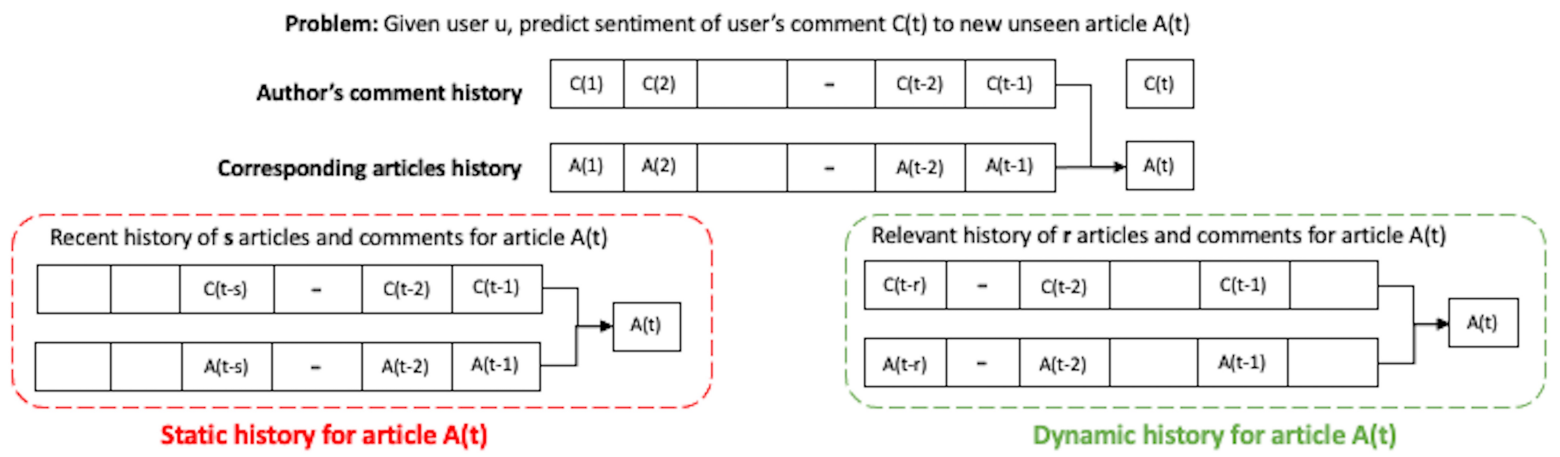

Figure 1: History selection techniques for dynamic and static fingerprints. In dynamic fingerprint, for a target article $\mathrm{A}(\mathrm{t})$ at time $\mathrm{t}, r$ relevant articles (in green) from user's history $\mathrm{A}(0)$ to $\mathrm{A}(\mathrm{t}-1)$ (in white) are selected based on semantic textual similarity between $\mathrm{A}(\mathrm{t})$ and history. In static fingerprint, $s$ articles (in red) from t-s to t- 1 time steps in immediate history are selected.

Target article: Pelosi Says House Will Condemn All Hate as Anti-Semitism Debate Overshadows Congress

\begin{tabular}{|c|c|c|}
\hline S No. & Static history & Dynamic history \\
\hline 1 & $\begin{array}{l}\text { Ilhan Omar Knows Exactly What She Is Do- } \\
\text { ing }\end{array}$ & $\begin{array}{l}\text { With Control of Congress at Stake, Trump } \\
\text { Reprises a Favorite Theme: Fear Immigrants }\end{array}$ \\
\hline 2 & $\begin{array}{l}\text { Ilhan Omar Controversy Caps a Month of } \\
\text { Stumbles for Democratic Leaders }\end{array}$ & $\begin{array}{l}\text { After Loss of House, Trump Makes Overture } \\
\text { to Democrats, Coupled With Threats }\end{array}$ \\
\hline 3 & Tom Brokaw: What Trump and Nixon Share & $\begin{array}{l}\text { Pompeo Speech Lays Out Vision for Mideast, } \\
\text { Taking Shots at Obama }\end{array}$ \\
\hline 4 & $\begin{array}{l}\text { Pelosi Says House Will Condemn All } \\
\text { Hate as Anti-Semitism Debate Overshadows } \\
\text { Congress }\end{array}$ & $\begin{array}{l}\text { White House Considers Using Storm Aid } \\
\text { Funds as a Way to Pay for the Border Wall }\end{array}$ \\
\hline 5 & Tariff Man Has Become Deficit Man & $\begin{array}{l}\text { Senate Leaders Plan Competing Bills to End } \\
\text { Shutdown }\end{array}$ \\
\hline 6 & $\begin{array}{l}\text { Paul Manafort to Be Sentenced Thursday in } 1 \\
\text { of } 2 \text { Cases Against Him }\end{array}$ & $\begin{array}{l}\text { House Votes to Block Trump's National Emer- } \\
\text { gency Declaration About the Border }\end{array}$ \\
\hline
\end{tabular}

Table 1: Articles extracted by different methods of history selection. We can see that dynamic history of articles are more relevant than static history.

are more related to the themes such as congress, hate etc., of target article. But static history does not show any relevant articles, except for article 4 , which was also because the user has read the target article in the recent past. So we can see that dynamic history has a more pertinent set of articles than static history.

\subsection{Text embedding}

In this stage, we create reading and writing tracks based on the selected user's history. Specifically, we encode articles and comments using contextual embedding by two types of encoders (BERT variants) - DistilBERT (Sanh et al., 2019) and ELECTRA (Clark et al., 2020) models. However, the proposed models are open to any variant of BERT encoder. We have used encoders in two modes - single-sentence mode where single span of contiguous text is encoded in form a special classification token $[C L S]$; two-sentence mode where two spans of text separated by $[S E P]$ token are encoded as $[C L S]$.

In the static FPE model, as shown in Figure 2, reading track comprises of selected articles, encoded by fixed-length $[C L S]$ tokens of single-sentence mode of BERT variant models, i.e., $\left[C L S_{t-s}^{R}, . ., C L S_{t-2}^{R}, C L S_{t-1}^{R}\right]$. We have conducted experiments both on - pretrained (with frozen parameters) and trained - BERT variants. Writing track comprises of both article and comments at each time step $\mathrm{k}\left[A_{k}, C_{i_{k}}\right]$, encoded as $[C L S]$ token outputs of two-sentence mode of BERT variant models, similar to reading track, i.e., 


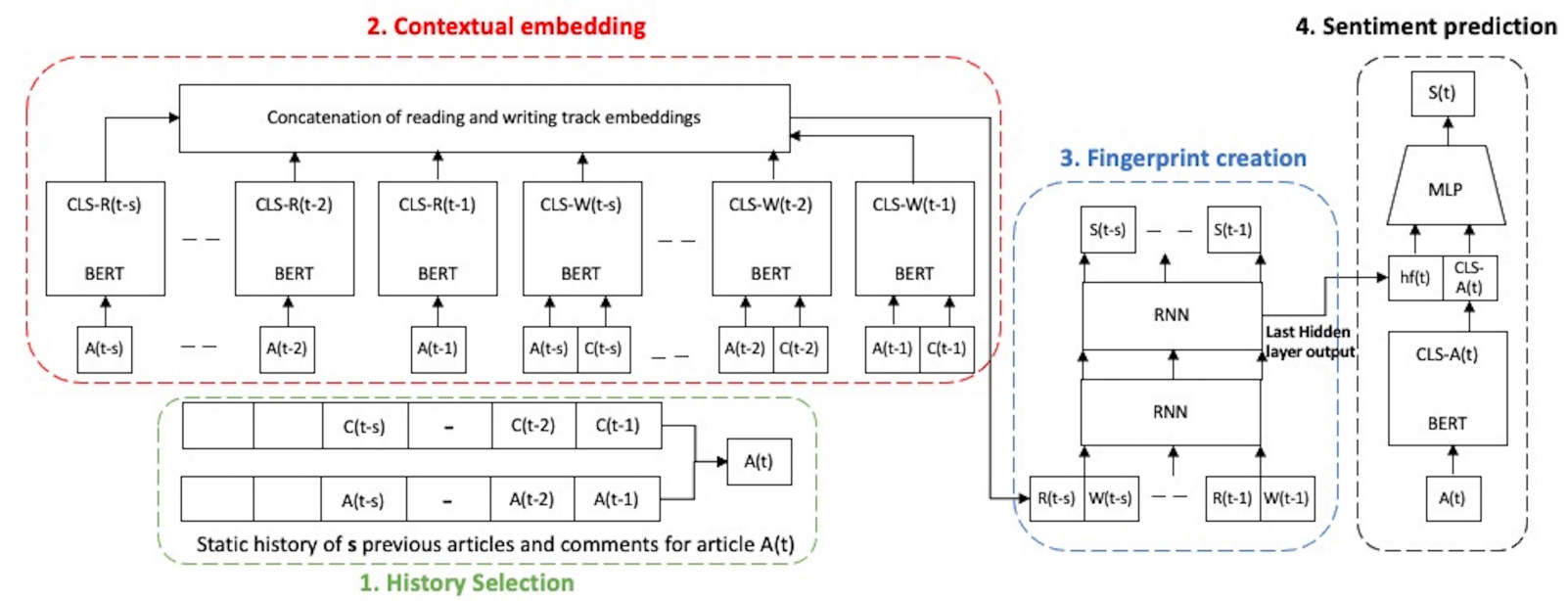

Figure 2: For the Static FPE model, in stage 1, for a target article A(t), $s$ article-comment pairs from (t-s) to (t-1) immediate past from a particular user's history are selected. In stage 2, the contextual embedding of $s$ articles are generated, and corresponding $[C L S]$ tokens from DistilBERT/ELECTRA models are extracted as reading track R(t-s) to R(t-1). Similarly, contextual embedding of articles A(t-s) to A(t-1) conditioned with corresponding comments $\mathrm{C}(\mathrm{t}-\mathrm{s})$ to $\mathrm{C}(\mathrm{t}-1)$ are generated and $[C L S]$ tokens are extracted as writing track $\mathrm{W}(\mathrm{t}-\mathrm{s})$ to $\mathrm{W}(\mathrm{t}-1)$. In stage 3, an RNN with 2 hidden layers is trained with concatenation of both tracks against corresponding comment sentiment $\mathrm{S}(\mathrm{t})$ at each time step $\mathrm{t}$ and the last hidden layer output is extracted as the fingerprint of user. In the last stage, a Multi-layer perceptron (MLP) is trained on the concatenation of the fingerprint $h_{t}^{f}$ and $C L S_{t}^{A}$ against the sentiment $\mathrm{S}(\mathrm{t})$ of the user's comment on article $\mathrm{A}(\mathrm{t})$.

$\left[C L S_{t-s}^{W}, . ., C L S_{t-2}^{W}, C L S_{t-1}^{W}\right]$

In the dynamic FPE model, as shown in Figure 3 , for the reading track - target article $A_{t}$ is appended to every article of relevant history and both of them are encoded by two-sentence mode of BERT variant models as $[C L S]$ token outputs, i.e., $\left[C L S_{t-r}^{R}, . ., C L S_{t-2}^{R}, C L S_{t-1}^{R}\right]$. The writing track is similar to that of static FPE model, i.e., $\left[C L S_{t-r}^{W}, . ., C L S_{t-2}^{W}, C L S_{t-1}^{W}\right]$.

Lastly, both the tracks are concatenated at each time step to create a unified fingerprint in case of static FPE model. But in dynamic FPE model, these tracks are used as separate entities to create two different fingerprints in the next stage.

\subsection{Fingerprint creation}

In this stage, unidirectional RNN is used to form contextual understanding of both reading and writing tracks.

In the static FPE model, each $[C L S]$ token of reading track is concatenated with corresponding $[C L S]$ token of writing track at each time step, i.e., $\left[C L S^{R}{ }_{t-s}, C L S^{W}{ }_{t-s} ; \ldots C L S_{t-2}^{R}, C L S^{W}{ }_{t-2}\right.$; $C L S_{t-1}^{R}, C L S_{t-1}^{W}$; ]. We have used Gated Recurrent Unit (GRU) (Chung et al., 2014) instead of LSTM, because the former has fewer parameters, trains faster with comparable performance to the latter. A two-layer GRU network is trained with the above concatenated output against corresponding sentiments $S_{t-s} \ldots, S_{t-2}, S_{t-1}$. The last hidden layer output is taken as the fingerprint embedding $h_{t}^{f}$ for article $A_{t}$ of user $u$.

In the Dynamic FPE model, we create a separate fingerprint for the reading track and writing track, respectively, with 2-layer RNN and 1-layer RNN networks. Both are GRU networks. An additional layer is used for the reading track to get a more complex feature representation of the relationship between target articles and the relevant history of articles. Here, fingerprint embedding is the concatenation of the last hidden layer outputs of both networks, i.e., $\left[h_{t}^{f_{R}} ; h_{t}^{f_{W}}\right]$.

\subsection{Sentiment prediction}

Lastly, a one-layer Multi-Layer Perceptron (MLP) is trained with concatenation of fingerprint embedding and $[C L S]$ token embedding of the target article, as input against the sentiment of response. In the static FPE model, the MLP is trained on $\left[h_{t}^{f} ; C L S_{t}^{A}\right]$ against output $S_{t}$. Whereas in the dynamic FPE model, it is trained on $\left[h_{t}^{f_{R}} ; h_{t}^{f_{W}} ; C L S_{t}^{A}\right]$ against output $S_{t}$.

\section{Experiments}

The main goals of our experiments are:

1. Measure the performance (in terms of micro 


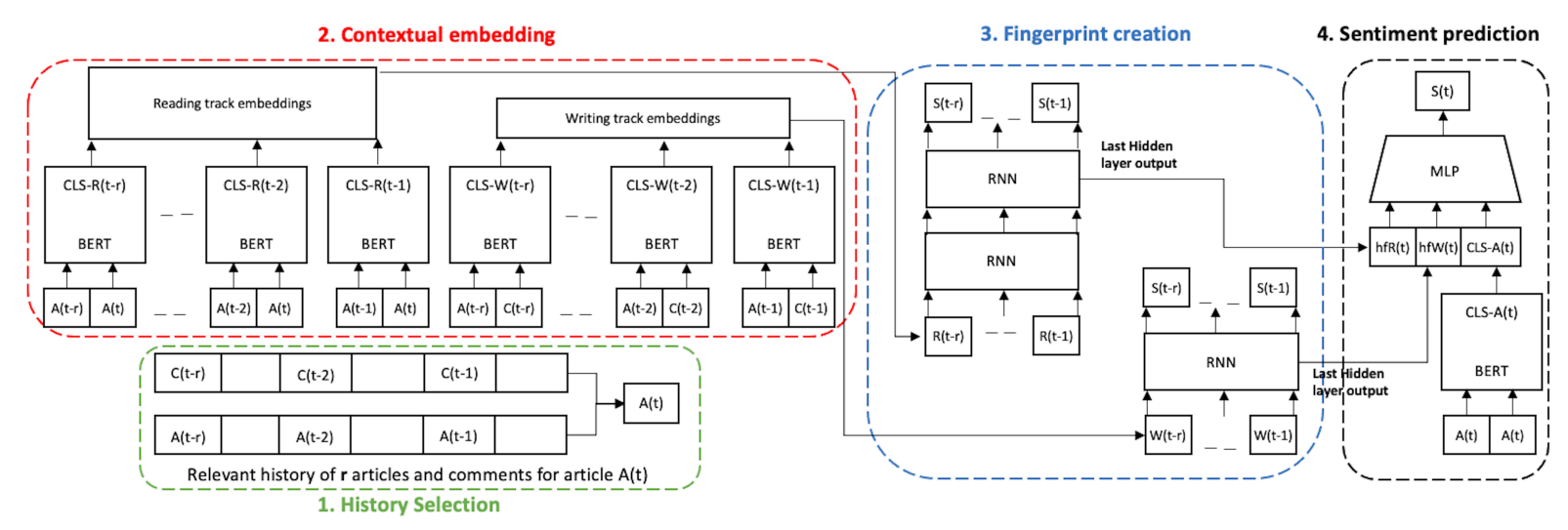

Figure 3: For the Dynamic FPE model, in stage 1, for a target article A(t), $r$ relevant article-comment pairs from a particular user's complete history are selected based on semantic textual similarity. In stage 2, the contextual embedding of each of the $r$ articles conditioned with target article $\mathrm{A}(\mathrm{t})$ is generated and corresponding $[C L S]$ tokens from DistilBERT/ELECTRA models are extracted as reading track. Similarly, the contextual embedding of articles $\mathrm{A}(\mathrm{t}-\mathrm{r})$ to $\mathrm{A}(\mathrm{t}-1)$ conditioned with corresponding comments $\mathrm{C}(\mathrm{t}-\mathrm{r})$ to $\mathrm{C}(\mathrm{t}-1)$ are generated, and $[C L S]$ tokens from DistilBERT/ELECTRA models are extracted as writing track. In stage 3, the reading track is encoded with a two-layered RNN trained against the corresponding comment sentiment $\mathrm{S}(\mathrm{t}-\mathrm{r})$ to $\mathrm{S}(\mathrm{t}-1)$ at each time step and the last hidden layer output is extracted as the reading fingerprint of the user. Similarly, the writing track is also encoded with a one-layered RNN and the last hidden layer output is extracted as a writing fingerprint. In the last stage, an MLP is trained on the concatenation of the reading fingerprint $h_{t}^{f_{R}}$, writing fingerprint $h_{t}^{f_{W}}$ and $C L S_{t}^{A}$ against sentiment $\mathrm{S}(\mathrm{t})$ of corresponding comment $\mathrm{C}(\mathrm{t})$ of user on article $\mathrm{A}(\mathrm{t})$.

F1-score) of both static and dynamic model variants in the prediction of the sentiment of a user to an unknown article

2. Analysis of model performance by studying the impact of dynamic history length and nature of articles on prediction.

\subsection{Data preparation}

We perform our empirical study on the datasets used for Personal opinion prediction by Yang et al., 2020.In these datasets, news articles are randomly selected from Archiveis, The Guardian, and New York Times. We do not consider users with fewer than ten comments. If after this step an article remains without any user, the article is discarded. We checked manually a random subset of articles and their comments and found that irrelevant comments are very few to ignore.

Each input example case comprises a target article/comment and its corresponding selected history of article-comment pairs. For each user, the data is split into training and test sets in the ratio of 90:10 ratio sequentially, i.e., the last $10 \%$ of comments made by user chronologically as test data and the remaining as training data. Also, the training set is split into training and validation data in the ratio of 90:10 preserving the sequential order.

Since our task is to predict the sentiment (as a score in $[-1,1])$ on a future comment, we considered 4 models to conceive the sentiment score. They are - Vader (Hutto and Gilbert, 2014), Flair (Akbik et al., 2019), BlobText sentiment, and BlobText subjectivity (Loria et al., 2014), to automatically label all comments. We assume that users have consistent views and stances on the same event within these articles and comments. Vader is a rule-based model for general sentiment analysis. It is constructed from a generalizable, valencebased, human-curated gold standard sentiment lexicon. When assessing the sentiment of tweets, Vader outperforms individual human raters (Hutto and Gilbert, 2014). Flair presents a unified interface for all word embeddings and supports methods for producing vector representation of entire documents. We use the Flair pre-trained classification model for sentiment labels. The model is trained on the IMDB dataset and has 90.54 micro F1-score. BlobText is a simple rule-based API for sentiment analysis. It has both sentiment model and subjectivity model, and we refer to them as Bsent and Bsubj respectively.

We used article titles and comment content as the basis for our model. We did not use article content since they are extremely long and moreover our focus is on user's opinion on the article, not the article per se. Table 2 shows the dataset statistics. 


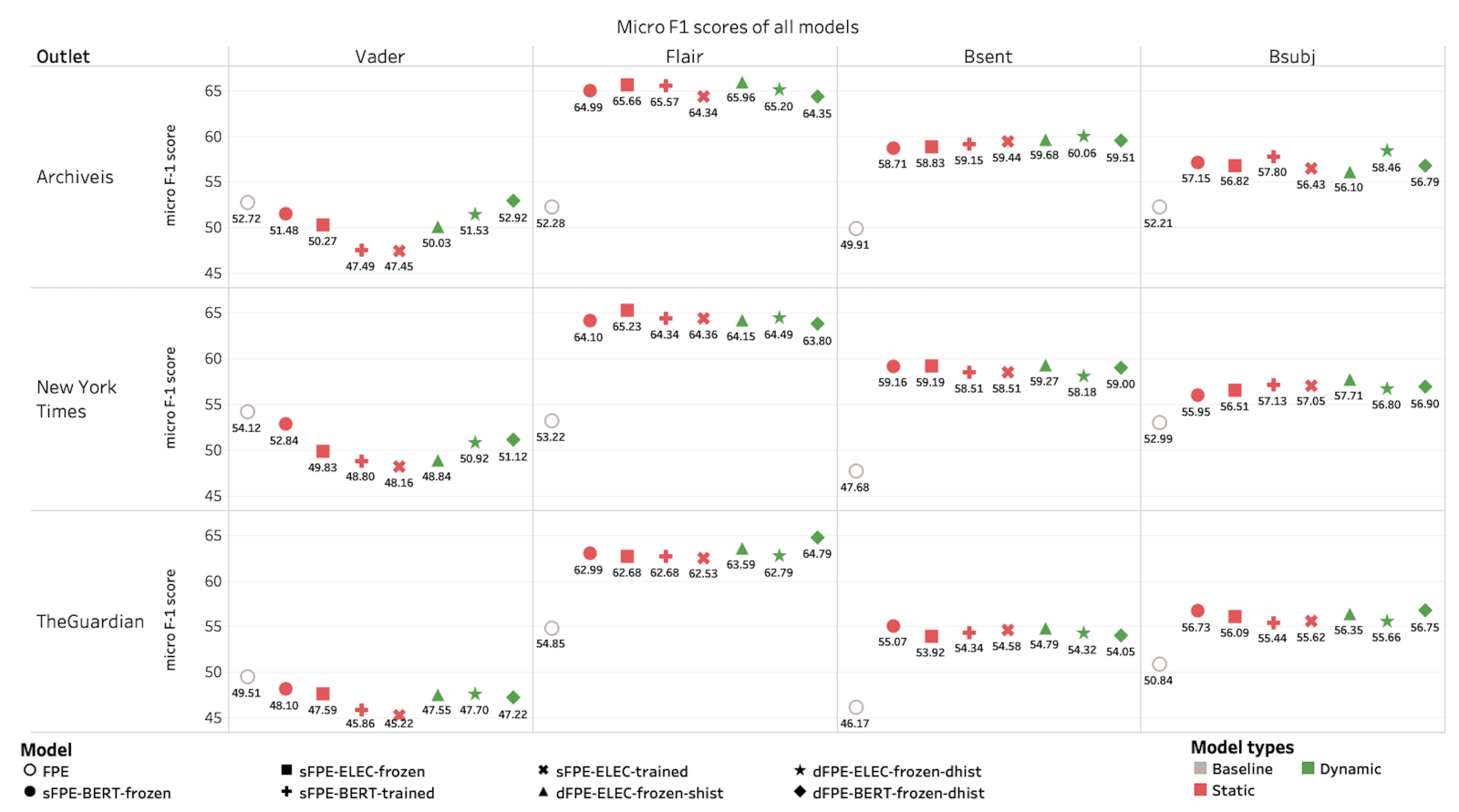

Figure 4: Micro F1-scores of all models on test data for all outlets with baseline scores, shown as white empty circle in the figure. We can see that dynamic FPE models have better performance of 4-13\% points over baseline FPE model, except for Vader sentiment, across all three outlets.

\begin{tabular}{llll}
\hline Statistics & Archiveis & TheGuardian & New YorkTimes \\
\hline$\# U$ & 20,920 & 41,069 & 37,957 \\
\hline \# Mean $C_{u}$ & 25.8 & 33.63 & 32.26 \\
\hline \# Med. $C_{u}$ & 9 & 13 & 10 \\
\hline$\# A$ & 2,043 & 6,393 & 3,647 \\
\hline$\# C$ & 812,768 & $5,467,755$ & $2,328,597$ \\
\hline
\end{tabular}

Table 2: Statistics for three news datasets. For each dataset, \# $U$ refers to total number of users, \# mean $C_{u}$ - mean number of comments per user, \# med. $C_{u}$ - median number of comments per user, \# $A$ - total number of articles and \# $C$ - total number of comments.

\subsection{Baselines and experiment settings}

We evaluated our models against the baseline Fingerprint embedding (FPE) model (Yang et al., 2020). In FPE, recent history of a target article was extracted and then Byte-Pair Embedding (BPE) (Heinzerling and Strube, 2018) and GRU were used to encode the words in articles and comments into fixed-length vectors. Subsequently, user's fingerprint was generated using a second GRU that modeled the sequence of history, which was a direct concatenation of prior articles and comments, encoded as fixed-length vectors. Finally, the concatenation of fingerprint embedding and target article embedding was given to MLP to predict the sentiment.

On the contrary, we examined both recent and relevant history of target article. We also used BERT based contextual embedding to encode the relationship between articles and comments rather than separate encoding. Finally, we created fingerprint separately for reading and writing track and then concatenated in the final stage for predicting the sentiment.

\subsection{Implementation}

As discussed in Section 3, we have primarily two models - static FPE and dynamic FPE- with different variants of each by using two types of contextual encoders- DistilBERT and ELECTRA, along with frozen and trained parameters. For static FPE models, we have taken an arbitrary history length of 12 article-comment pairs, so $s=12$ for fingerprinting. For dynamic FPE models, we have taken relevant dynamic history length of 15 articlecomment pairs, $r=15$, after comparing micro F1-scores for various lengths from 5 to 20 . We discuss about the impact of dynamic history length on performance in Section 5. In all cases, a hidden layer of GRU with a dimension of 256 is created. We have trained all model variants for 10 epochs and saved the model based on the mean micro F1score over all four sentiments on the validation dataset. Usually, the best model is achieved around 5-6 epochs. 


\begin{tabular}{|c|c|c|c|}
\hline Model & Type & $\begin{array}{ll}\text { BERT } & \text { history } \\
\text { (param- } & \text { type } \\
\text { eters) } & \end{array}$ & $\begin{array}{l}\text { history } \\
\text { length }\end{array}$ \\
\hline $\begin{array}{l}\text { SFPE- } \\
\text { BERT- } \\
\text { frozen }\end{array}$ & $\begin{array}{l}\text { static } \\
\text { FPE }\end{array}$ & $\begin{array}{l}\text { DistilBERT static } \\
\text { (frozen) }\end{array}$ & 12 \\
\hline $\begin{array}{l}\text { SFPE- } \\
\text { BERT- } \\
\text { trained }\end{array}$ & $\begin{array}{l}\text { static } \\
\text { FPE }\end{array}$ & $\begin{array}{l}\text { DistilBERT static } \\
\text { (trained) }\end{array}$ & 12 \\
\hline $\begin{array}{l}\text { sFPE- } \\
\text { ELEC- } \\
\text { frozen }\end{array}$ & $\begin{array}{l}\text { static } \\
\text { FPE }\end{array}$ & $\begin{array}{l}\text { ELECTRA static } \\
\text { (frozen) }\end{array}$ & 12 \\
\hline $\begin{array}{l}\text { sFPE- } \\
\text { ELEC- } \\
\text { trained }\end{array}$ & $\begin{array}{l}\text { static } \\
\text { FPE }\end{array}$ & $\begin{array}{l}\text { ELECTRA static } \\
\text { (trained) }\end{array}$ & 12 \\
\hline $\begin{array}{l}\text { dFPE- } \\
\text { ELEC- } \\
\text { frozen- } \\
\text { shist }\end{array}$ & $\begin{array}{l}\text { dynamic } \\
\text { FPE }\end{array}$ & $\begin{array}{l}\text { ELECTRA static } \\
\text { (frozen) }\end{array}$ & 12 \\
\hline $\begin{array}{l}\text { dFPE- } \\
\text { ELEC- } \\
\text { frozen- } \\
\text { dhist }\end{array}$ & $\begin{array}{l}\text { dynamic } \\
\text { FPE }\end{array}$ & $\begin{array}{l}\text { ELECTRA dynamic } \\
\text { (frozen) }\end{array}$ & 15 \\
\hline $\begin{array}{l}\text { dFPE- } \\
\text { BERT- } \\
\text { frozen- } \\
\text { dhist }\end{array}$ & $\begin{array}{l}\text { dynamic } \\
\text { FPE }\end{array}$ & $\begin{array}{l}\text { DistilBERT dynamic } \\
\text { (frozen) }\end{array}$ & 15 \\
\hline
\end{tabular}

Table 3: Notation of models along with their configuration details about type of FPE model, BERT variant, history type, length of history.

We use Adam optimizer with weight decay (Loshchilov and Hutter, 2019) and a schedule of learning rate (lr) that decreases following the values of the cosine function between the initial $\mathrm{lr}$ set in the optimizer to 0 , with several hard restarts, after a warmup period during which it increases linearly between 0 and the initial lr set to 0.001 in the optimizer. We implemented the model using PyTorch lightning, a wrapper for PyTorch. The code is released at GitHub.

\section{Results and discussion}

Table 3 describes the model notation and corresponding configuration used in the results. Figure 4 shows that except for vader sentiment, all our model variants outperform the FPE baseline. In the remaining three sentiments, dynamic-FPEELECTRA (frozen) model with either dynamic or static history outperformed the remaining variants and also FPE baseline. This could be because of multiple reasons, and we discuss them below.

Firstly, contextual embedding, in place of static BPE embedding in FPE, of articles and comments is a key factor behind the superior performance. Specifically, the contextual embedding of article history with target article (reading track) and with corresponding comment history (writing track) has enabled us to generate a better representation of the input text.

Secondly, the dynamic FPE model, unlike the static FPE model, creates reading and writing fingerprints through GRU networks separately before concatenating them for sentiment prediction. With an extra GRU hidden layer in the reading track compared to the writing track, we have been able to create a higher level of understanding of the temporal relationship between target article and history articles. From these fingerprints, we also found that users with the closest fingerprints in the euclidean space are found to have higher prediction accuracy than that of farther fingerprint users.

Further, the ELECTRA-based model outperforms DistilBERT-based model in most of the cases, despite having only $20 \%$ of the number of parameters of DistilBERT-based model. This reiterates the result of Clark et al., 2020 that novel pretraining by a discriminative model that predicts whether each token in the corrupted input was replaced by a generator sample or not performs better than masked language modeling pretraining method of DistilBERT, even for a small model.

Other important inferences are:

1. Performance increases with length of relevant history for dynamic FPE models in general;

2. Articles with a large proportion of comments with negative sentiment have a higher micro F1-score compared to controversial (equally positive and negative sentiment comments) and positive comments (predominant positive sentiment comments).

3. BERT variant models with frozen parameters have better performance than those with all parameters trained. This may be because the pretrained model is trained on a much larger corpus than our problem dataset and so it has better language understanding.

For complete details of these experiments, please refer to longer version of the paper .

\section{Limitations and future work}

According to Sutrop and Laas-Mikko, 2012, fingerprinting for predicting behavior is secondgeneration biometrics, which is different from firstgeneration biometrics that uses characteristics that are visible to the naked eye such as facial images, 
hand fingerprints. In this context, user fingerprinting in our model can be loosely classified under behavior fingerprinting. We did linguistic opinion and content-based user fingerprinting as a response history embedding for a user. In this section, we briefly discuss the limitations of the dataset and model, and future research direction.

Firstly, the newspaper articles, in general, may be biased in terms of story selection, tone, and organizing of the story. The users(readers) may also have an implicit bias - attitudes that unconsciously affect individual thoughts and actions- and confirmation bias - the tendency to support information that confirms their beliefs. To address these biases, we would like to extend our model to datasets that are not related to news articles.

Moreover, representations encoded in the models often inadvertently perpetuate undesirable social biases from the data on which they are trained. NLP models, especially neural embeddings, may perpetuate these biases towards race, religion, gender and disability (Hutchinson et al., 2020; Manzini et al., 2019; Sap et al., 2019). Though the BERT variant based sentence encoders exhibit less bias than previous models (May et al., 2019), we would also like to experiment with other sentence encoders to measure the bias in our predictions in future work.

Another limitation of our approach is that we used only article titles rather than whole content. This would be more critical when the title is misleading, for instance in satirical articles. Moreover, we have not experimented with multilingual models of pretrained BERT variants.

For future research, these experiments can be extended for whole article content and use various at tention mechanisms to generate better fingerprints and also generate author profiles based on their reading history. Further, BERT variants trained in other languages can also be used.

\section{Conclusion}

In this paper, we propose a novel dynamic fingerprinting technique based on BERT variants and RNN networks to predict a user's sentiment to an unseen article based on reading-writing history. Two variants of our model extract relevant history in two different ways and create contextual embedding for articles read by a user conditioned with target article and also corresponding comments. Finally, we used RNN to interpret temporal relationship and create a fingerprint, which is used to predict unseen target article. Our models demonstrated state-of-the-art performance on a real-world dataset. From our experiments, we found that performance saturates after an optimum length of relevant history.

\section{Acknowledgments}

Research was supported in part by grants NSF 1838147, NSF 1838145, ARO W911NF-20-10254. The views and conclusions contained in this document are those of the authors and not of the sponsors. The U.S. Government is authorized to reproduce and distribute reprints for Government purposes notwithstanding any copyright notation herein. Finally, we are greatly appreciative of the anonymous reviewers for their time and constructive comments.

\section{References}

Alan Akbik, Tanja Bergmann, Duncan Blythe, Kashif Rasul, Stefan Schweter, and Roland Vollgraf. 2019. FLAIR: an easy-to-use framework for state-of-theart NLP. In Proceedings of the 2019 Conference of the North American Chapter of the Association for Computational Linguistics: Human Language Technologies, NAACL-HLT 2019, Minneapolis, MN, USA, June 2-7, 2019, Demonstrations, pages 54-59. Association for Computational Linguistics.

Josemar Alves Caetano, Helder Seixas Lima, Mateus F. Santos, and Humberto Torres Marques-Neto. 2018. Using sentiment analysis to define twitter political users' classes and their homophily during the 2016 american presidential election. J. Internet Serv. Appl., 9(1):18:1-18:15.

Junyoung Chung, Çaglar Gülçehre, KyungHyun Cho, and Yoshua Bengio. 2014. Empirical evaluation of gated recurrent neural networks on sequence modeling. CoRR, abs/1412.3555.

Kevin Clark, Minh-Thang Luong, Quoc V. Le, and Christopher D. Manning. 2020. ELECTRA: pretraining text encoders as discriminators rather than generators. CoRR, abs/2003.10555.

Jacob Devlin, Ming-Wei Chang, Kenton Lee, and Kristina Toutanova. 2019. BERT: pre-training of deep bidirectional transformers for language understanding. In Proceedings of the 2019 Conference of the North American Chapter of the Association for Computational Linguistics: Human Language Technologies, NAACL-HLT 2019, Minneapolis, MN, USA, June 2-7, 2019, Volume 1 (Long and Short Papers), pages 4171-4186. Association for Computational Linguistics. 
Benjamin Heinzerling and Michael Strube. 2018. Bpemb: Tokenization-free pre-trained subword embeddings in 275 languages. In Proceedings of the Eleventh International Conference on Language Resources and Evaluation, LREC 2018, Miyazaki, Japan, May 7-12, 2018. European Language Resources Association (ELRA).

Ben Hutchinson, Vinodkumar Prabhakaran, Emily Denton, Kellie Webster, Yu Zhong, and Stephen Denuyl. 2020. Social biases in NLP models as barriers for persons with disabilities. In Proceedings of the 58th Annual Meeting of the Association for Computational Linguistics, ACL 2020, Online, July 5-10, 2020, pages 5491-5501. Association for Computational Linguistics.

Clayton J. Hutto and Eric Gilbert. 2014. VADER: A parsimonious rule-based model for sentiment analysis of social media text. In Proceedings of the Eighth International Conference on Weblogs and Social Media, ICWSM 2014, Ann Arbor, Michigan, USA, June 1-4, 2014. The AAAI Press.

Lei Li, Yabin Wu, Yuwei Zhang, and Tianyuan Zhao. 2019. Time+user dual attention based sentiment prediction for multiple social network texts with time series. IEEE Access, 7:17644-17653.

Ilya Loshchilov and Frank Hutter. 2019. Decoupled weight decay regularization. In 7 th International Conference on Learning Representations, ICLR 2019, New Orleans, LA, USA, May 6-9, 2019. OpenReview.net.

Thomas Manzini, Yao Chong Lim, Alan W. Black, and Yulia Tsvetkov. 2019. Black is to criminal as caucasian is to police: Detecting and removing multiclass bias in word embeddings. In Proceedings of the 2019 Conference of the North American Chapter of the Association for Computational Linguistics: Human Language Technologies, NAACL-HLT 2019, Minneapolis, MN, USA, June 2-7, 2019, Volume 1 (Long and Short Papers), pages 615-621. Association for Computational Linguistics.

Chandler May, Alex Wang, Shikha Bordia, Samuel R. Bowman, and Rachel Rudinger. 2019. On measuring social biases in sentence encoders. In Proceedings of the 2019 Conference of the North American Chapter of the Association for Computational Linguistics: Human Language Technologies, NAACLHLT 2019, Minneapolis, MN, USA, June 2-7, 2019, Volume 1 (Long and Short Papers), pages 622-628. Association for Computational Linguistics.

Yasuhito Ohsugi, Itsumi Saito, Kyosuke Nishida, Hisako Asano, and Junji Tomita. 2019. A simple but effective method to incorporate multi-turn context with BERT for conversational machine comprehension. CoRR, abs/1905.12848.

Nils Reimers and Iryna Gurevych. 2019. Sentencebert: Sentence embeddings using siamese bertnetworks. In Proceedings of the 2019 Conference on
Empirical Methods in Natural Language Processing and the 9th International Joint Conference on Natural Language Processing, EMNLP-IJCNLP 2019, Hong Kong, China, November 3-7, 2019, pages 3980-3990. Association for Computational Linguistics.

Victor Sanh, Lysandre Debut, Julien Chaumond, and Thomas Wolf. 2019. Distilbert, a distilled version of BERT: smaller, faster, cheaper and lighter. CoRR, abs/1910.01108.

Maarten Sap, Dallas Card, Saadia Gabriel, Yejin Choi, and Noah A. Smith. 2019. The risk of racial bias in hate speech detection. In Proceedings of the 57th Conference of the Association for Computational Linguistics, ACL 2019, Florence, Italy, July 28-August 2, 2019, Volume 1: Long Papers, pages 16681678. Association for Computational Linguistics.

Jianfeng Si, Arjun Mukherjee, Bing Liu, Qing Li, Huayi Li, and Xiaotie Deng. 2013. Exploiting topic based twitter sentiment for stock prediction. In Proceedings of the 51st Annual Meeting of the Association for Computational Linguistics, ACL 2013, 4-9 August 2013, Sofia, Bulgaria, Volume 2: Short Papers, pages 24-29. The Association for Computer Linguistics.

Margit Sutrop and Katrin Laas-Mikko. 2012. From identity verification to behavior prediction: Ethical implications of second generation biometrics. Review of Policy Research, 29(1):21-36.

Fan Yang, Eduard C. Dragut, and Arjun Mukherjee. 2020. Predicting personal opinion on future events with fingerprints. In Proceedings of the 28th International Conference on Computational Linguistics, COLING 2020, Barcelona, Spain (Online), December 8-13, 2020, pages 1802-1807. International Committee on Computational Linguistics.

Chenguang Zhu, Michael Zeng, and Xuedong Huang. 2018. Sdnet: Contextualized attention-based deep network for conversational question answering. CoRR, abs/1812.03593. 